EPJ Web of Conferences 66, 02038 (2014)

DOI: $10.1051 /$ epjconf/ 201466032038

(C) Owned by the authors, published by EDP Sciences, 2014

\title{
Microscopic optical potential with two and three body forces for nucleon - nucleus scattering
}

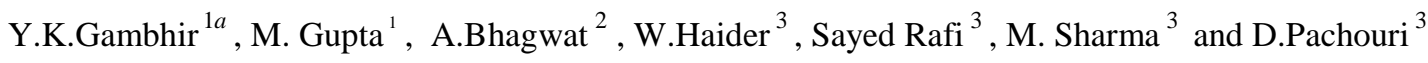 \\ ${ }^{1}$ MCNS, Manipal University, Manipal 576 104, India \\ ${ }^{2}$ EUM - DAE, CBS, Mumbai 400 098, India \\ ${ }^{3}$ Department of Physics, AMU, Aligarh 200002
}

\begin{abstract}
The proton - nucleus optical potentials generated by folding the calculated complex, density and energy dependent g- matrices (with and without three-body forces (TBF): Urbana IX (UVIX) and TNI) over the target nucleon density distributions obtained from the relativistic mean field theory, are used for the calculation of the differential cross section $d \sigma / d \theta$, polarization $A_{y}$, spin rotation function (Q). for 65 and $200 \mathrm{MeV}$ polarized proton incident on ${ }^{40} \mathrm{Ca}$ and ${ }^{208} \mathrm{~Pb}$. The agreement with the experiment is rather impressive. It is found that the inclusion of TBF (Urbana IX (UVIX) and TNI) reduces the strength of the central part of the optical potential in the nuclear interior and affects the calculated spin-orbit potential only marginally and leads to an improvement in the agreement with the corresponding experimental results.
\end{abstract}

\section{Introduction}

The nucleon - nucleus ( $\mathrm{n}$ - A) scattering serves a useful tool to study nuclear properties .The observables are the differential cross section $(d \sigma / d \theta)$, analyzing power or asymmetry $\left(A_{y}\right)$ and the spin rotation function $(Q)$. The Brueckner - Hartree - Fock (BHF) based microscopic optical potential (OP) used in the calculation of these observables requires only two inputs: (1) The nucleon - nucleon $(\mathrm{N}-\mathrm{N})$ interaction to calculate the reaction or $\mathrm{g}$ - matrix and (2) the nucleon density distributions of target nuclei to generate the optical potential.

\section{Details of the calculation}

The three body force (TBF) is believed to be essential in correctly reproducing the saturation properties of symmetric nuclear matter - as all the available realistic two body interactions fail. Here, we use high quality modern local soft core N - N potential Argonne v18 [1] (AV18) with and without two models of three nucleon force: (1) the Urbana IX [2] (UV IX) and (2) the three nucleon interaction model TNI $[3,4]$, obtained in the BHF. We fold the appropriate numerically computed complex reaction matrices (as defined in [5]) over the proton and the neutron density distributions of the target, using the local density approximation to obtain both the real and imaginary parts of the

${ }^{a}$ yogy@phy.iitb.ac.in

This is an Open Access article distributed under the terms of the Creative Commons Attribution License 2.0, which permits unrestricted use, distribution, and reproduction in any medium, provided the original work is properly cited. 
central as well as of the spin - orbit components of the proton - nucleus optical potential. The details of the calculations and the self consistency are described in detail in [5,6].
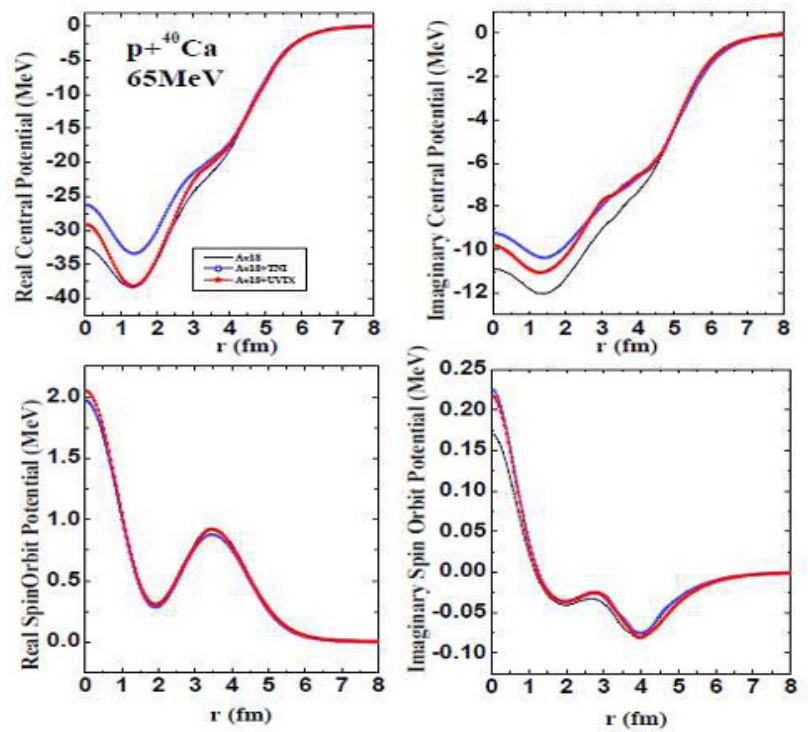

Figure 1: The calculated central and spin-orbit parts of the optical potential for $\mathrm{p}-{ }^{40} \mathrm{Ca}$ scattering at $65 \mathrm{MeV}$.

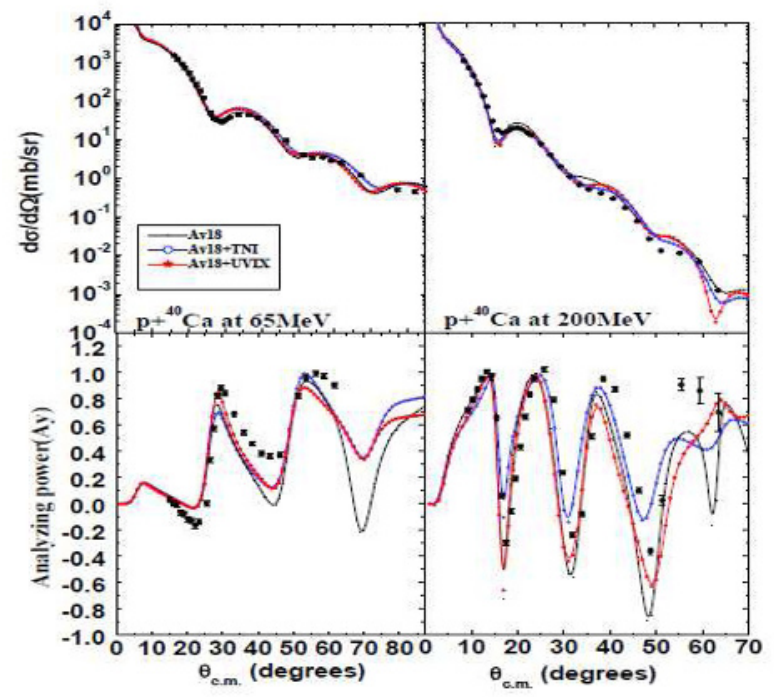

Figure 2: The differential cross section and the analyzing power for $\mathrm{p}-{ }^{40} \mathrm{Ca}$ scattering.

\section{Results, discussion and conclusion}

It is observed that the inclusion of TBF (UVIX and TNI) : Reduces both the real and imaginary parts of the central parts of the potential in the interior region $(r<5 \mathrm{fm})$ of the target at both the energies 
considered here and only, marginally effects the spin - orbit parts of the optical potential (Fig. 1: Illustration for $\mathrm{p}-{ }^{40} \mathrm{Ca}$ scattering at $65 \mathrm{MeV}$ ). Identical trends are observed for $\mathrm{p}-{ }^{208} \mathrm{~Pb}$ scattering. The numerically computed complex central and the spin - orbit parts of the OP are used in the optical model code to calculate the total and differential cross sections $(d \sigma / d \theta)$, asymmetry or polarization $\left(A_{y}\right)$ (Figs. 2 and 3) and the spin rotation function (Q) (Fig. 4) for p - ${ }^{40} \mathrm{Ca}$ and p ${ }^{208} \mathrm{~Pb}$ scattering at 65 and $200 \mathrm{MeV}$ proton energies. Three types of $\mathrm{g}$ - matrices calculated by using AV18, AV18 + UVIX and AV18 + TNI, are folded over the nucleon density distributions of the

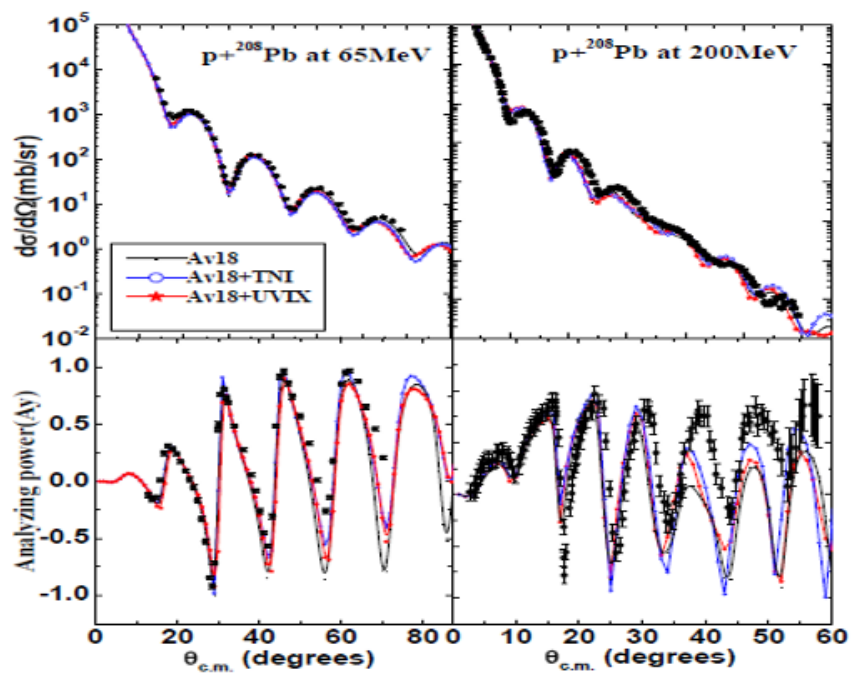

Figure 3: The differential cross section and the analyzing power for $\mathrm{p}-{ }^{208} \mathrm{~Pb}$ scattering.

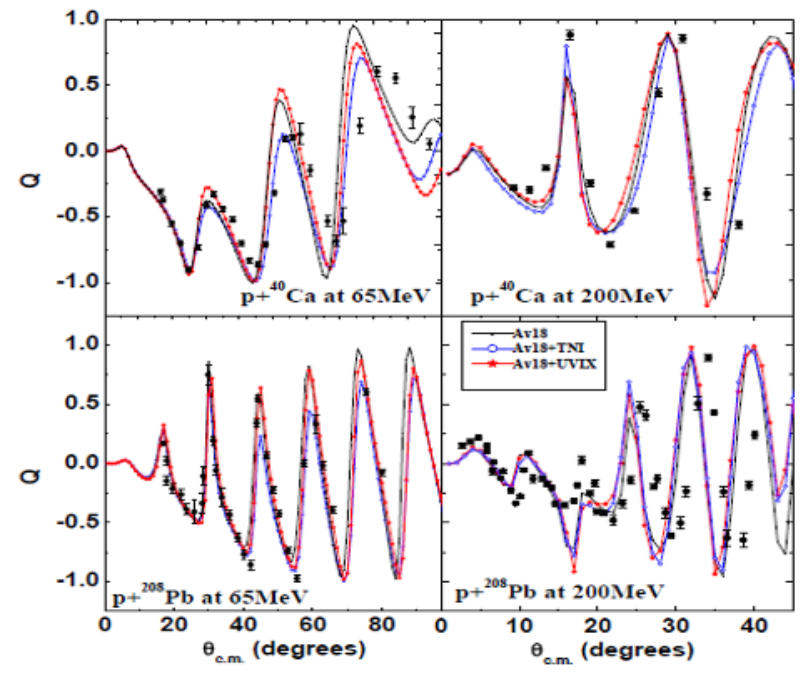

Figure 4: The spin rotation function (Q) for $\mathrm{p}-{ }^{40} \mathrm{Ca}$ and $\mathrm{p}-{ }^{208} \mathrm{~Pb}$ scattering. 
respective targets, to generate the corresponding central and spin - orbit parts of the complex optical potentials. To obtain better agreement with the experiment, we follow the usual practice and scale the calculated imaginary parts of the OP by a factor 0.7 and the real central part of the optical potential at $200 \mathrm{MeV}$ by a factor of 1.2. These scaling factors are determined through the chi-squares fit to the experimental data. The agreement between the calculation and the corresponding experimental results is rather impressive. Further, the inclusion of TBF (UVIX and TNI) improves the quality of the agreement with the experiment as is evident from the calculated $\chi^{2} / D F$ per degrees of freedom (listed in Table - I for both the targets at both the energies considered here.)

Table 1: The $\chi_{X}^{2} / D F: X=\sigma$, pol, Q and tot represent the differential cross section, analyzing power, spin rotation and total cross section respectively for the experimental data analyzed.

\begin{tabular}{|c|c|c|c|c|}
\hline & & AV18 & AV18+UVIX & AV18+TNI \\
\hline $\mathbf{p}-{ }^{40} \mathrm{Ca}$ & $\chi_{\sigma}^{2} / D F$ & 7.41 (70.59) & 7.89 (21.39) & $3.61(38.8)$ \\
\hline \multirow[t]{3}{*}{65 (200) MeV } & $\chi_{p o l}^{2} / D F$ & $53.52(15.85)$ & $36.15(10.98)$ & $25.49(12.48)$ \\
\hline & $\chi_{Q}^{2} / D F$ & $39.76(24.81)$ & $26.25(29.56)$ & 30.98 \\
\hline & $\chi_{t o t}^{2} / D F$ & $32.55(40.15)$ & $22.79(18.40)$ & $19.42(25.09)$ \\
\hline $\mathbf{p}-{ }^{208} \mathrm{~Pb}$ & $\chi_{\sigma}^{2} / D F$ & $26.94(37.61)$ & $22.03(23.40)$ & $15.04(21.24)$ \\
\hline \multirow[t]{3}{*}{65 (200) MeV } & $\chi_{p o l}^{2} / D F$ & $16.48(46.65)$ & $20.17(24.77)$ & $9.51(19.80)$ \\
\hline & $\chi_{Q}^{2} / D F$ & $36.27(13.06)$ & $28.63(9.03)$ & $13.83(7.78)$ \\
\hline & $\chi_{t o t}^{2} / D F$ & $29.73(92.39)$ & $14.83(64.97)$ & $26.75(66.96)$ \\
\hline
\end{tabular}

\section{References}

1. R. B. Wiringa, V. G. J. Stokes, R. Schiavilla, Phys. Rev. C 51, 38 (1995)

2. B. S. Pudliner et. al. Phys. Rev. Lett. 74, 4396 (1995); Phys. Rev. C 56, 1720 (1997)

3. I. E. Lagris, V. R. Pandharipande, Nucl. Phys. A 359, 349 (1981)

4. B. Friedman, V. R. Pandharipande, Nucl. Phys. A 361, 502 (1981)

5. S. M. Saliem, W. Haider, J. Phys. G 28, 1313 (2002)

6. Syed Rafi, Manjari Sharma, Dipti Pachouri, W. Haider, Y. K. Gambhir Phys. Rev. C 87, 014003 (2013) and references cited there in 